\title{
Computation of Buckling Pressure of Pumpkin Balloons
}

\author{
M. Pagitz*and S. Pellegrino ${ }^{\dagger}$ \\ University of Cambridge, Cambridge, CB2 1PZ, UK
}

\begin{abstract}
This paper is concerned with the elastic buckling of pumpkin-shaped balloon structures. Pumpkin balloons, currently being used in the NASA Ultra-Long Duration Ballooning (ULDB) Program, consist of stiff meridional tendons that constrain a thin, highly curved plastic surface. The present study exploits the high degree of symmetry of this design by adopting a newly developed, closed form expression of a symmetry-adapted coordinate system for structures with $n$-fold symmetry, which provides the tangent stiffness matrix in a very efficient block-diagonal form. The critical buckling pressure of balloons with different properties are computed. It is found that for any chosen balloon there is a power law relation between the critical pressure and the number of lobes. The change in the powerlaw coefficients is used to study the sensitivity of different balloons to design parameters such as the shape of the lobe cutting pattern and the elastic properties of the membrane and tendons.
\end{abstract}

\section{Introduction and Background}

The beginning of modern scientific ballooning dates back to the early 1950's with the advent of the first zero-superpressure balloons made out of thin polyethylene foil. These balloons are lobed and open at the bottom, like hot-air balloons. However, zero-superpressure balloons are not able to keep a constant altitude during a mission. The reason is that the helium inside the balloon is periodically heated and cooled during the day-night cycle, which leads to volume changes. In order to keep the volume constant, it is necessary to have a closed envelope where the day-time differential pressure is set sufficiently high that at night there still is some residual pressure.

Pumpkin (superpressure) balloons have a closed envelope and are subject to a sufficiently large differential pressure that they are able to stay at constant altitude. Such a design was first considered by G.I. Taylor ${ }^{1}$ in 1919 during a study of the shapes of parachutes, Figure 1. However, the pumpkin shape, although attractive in terms of pressure-carrying efficiency has been found to be unstable.

The first construction of a large scale pumpkin balloon dates back to 1984 when J. Nott designed the Endeavour super-pressure balloon, to circumnavigate the world. When first inflated, Nott's 64 lobe pumpkin balloon $^{2,3}$ adopted a non-symmetrical shape, even at low differential pressure, and remained heavily asymmetric at higher pressure, Figure 2. Removal of two lobes diminished, but not eliminated, the asymmetry and finally removal of a total of four lobes led to a symmetrical balloon.

Calladine $^{3}$ explained the buckling phenomenon by showing that the volume enclosed by a lobed balloon whose lobes subtend a constant angle increases - if the number of lobes and the bulge formed by the lobes are sufficiently large - for certain small-amplitude inextensional deformations of the balloon. Later, Lennon and Pellegrino ${ }^{4}$ showed that - again for inextensional deformation modes - the volume enclosed by a constant lobe radius balloon decreases for small deformations but increases for sufficiently large deformations. However, both of these analytical models are based on assumed buckling modes and neglect the elastic deformation of the membrane and tendons.

In the 1990s NASA started to develop an Ultra Long Duration Balloon (ULDB) ${ }^{5}$ to support global scientific observations above $99 \%$ of the earth's atmosphere. These balloons are intended to carry a payload of several tonnes for periods approaching 100 days. To realise this ambitious goal NASA has chosen a pumpkin

\footnotetext{
*Research student, Department of Engineering, Trumpington Street.

${ }^{\dagger}$ Professor of Structural Engineering, Department of Engineering, Trumpington Street. Associate Fellow AIAA.
} 


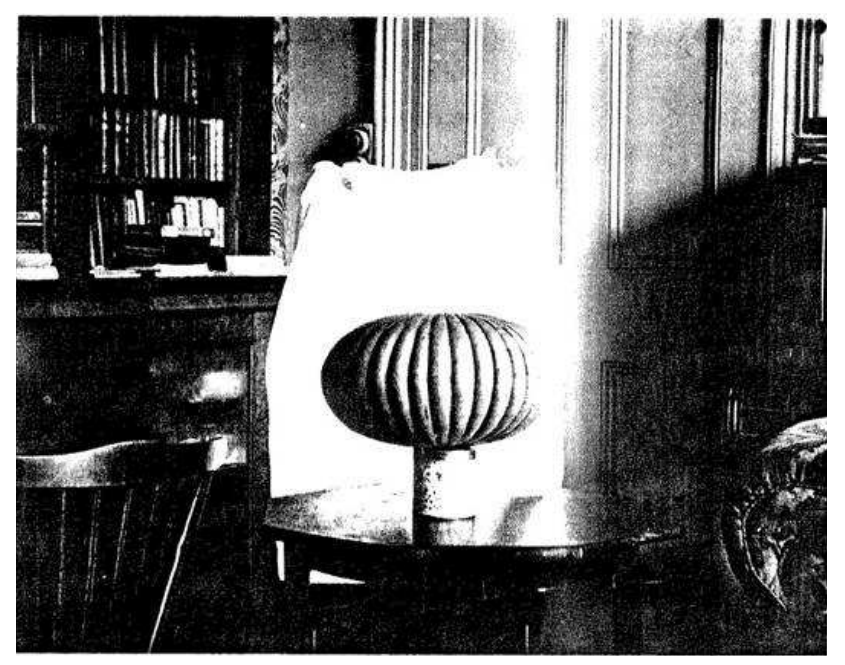

Figure 1. Pumpkin balloon made by G.I. Taylor. ${ }^{1}$

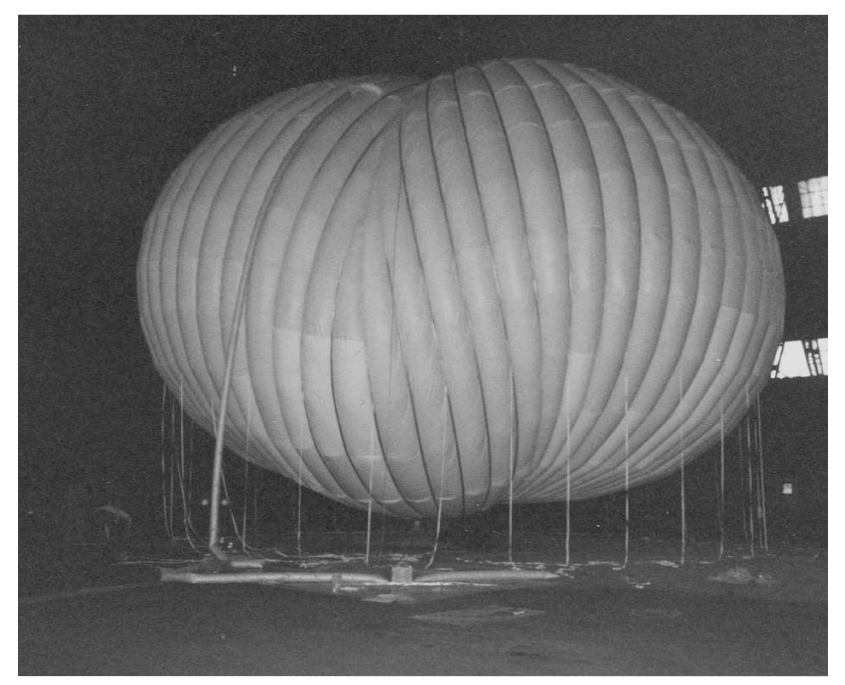

Figure 2. Unstable pumpkin balloon (courtesy of J. Nott). 
balloon design made out of state of the art materials. Nevertheless the same instability encountered in the Endeavour balloon has also affected the initial stages of this development, ${ }^{6}$ and this has recently prompted the development of scale models to fully understand this behaviour. ${ }^{7,8}$ Results from some of these experiments will be used for validating the numerical simulations presented in this paper.

In this paper we extend the previous work of Refs. ${ }^{3,4}$ to formulate a detailed numerical model of a pumpkin balloon which fully accounts for the shape and elasticity of the lobes, and yet is compact enough that it can be used to study a large number of designs in order to pick out general trends of this complex structure.

\section{Concept of Pumpkin Balloon}

Pumpkin balloons are assembled from $n$ identical lobes, Figure 3. If we consider the surface of an inflated lobe then it is obvious that its doubly-curved surface is not developable. However, it would be impractical to make the lobes from non-flat sheets, and so each lobe is manufactured according to a specified cuttingpattern, from a flat sheet. Hence, the only way of controlling the shape of the lobe is through the edge profile of the lobe (note that material anisotropy could also be exploited).

The structure is assembled by welding neighbouring lobes together and incorporating a stiff tendon along the common boundary. As it is not possible to terminate the lobes directly at the apexes, end-fittings are used. An end-fitting consists of two circular plates that seal the balloon and a ring to which the tendons are attached.

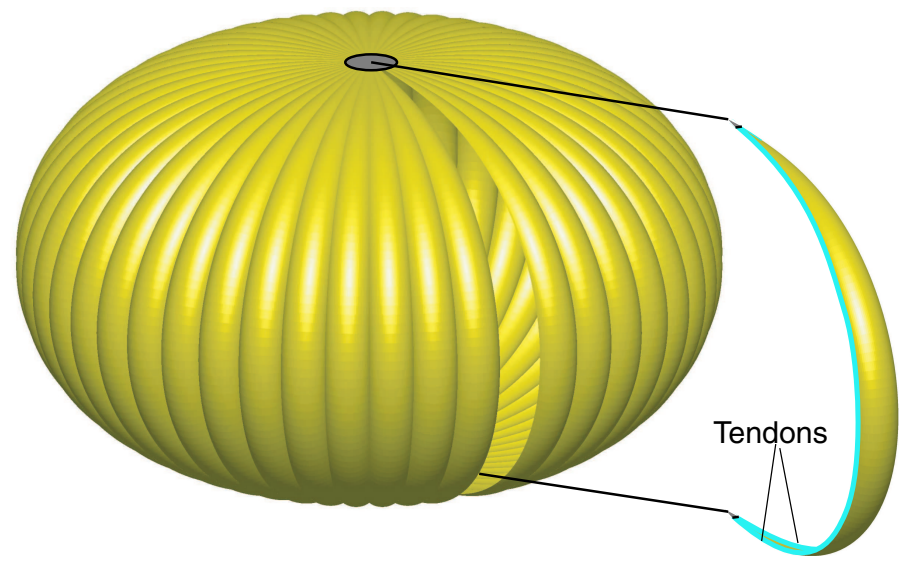

Figure 3. Schematic drawing of 48 lobe pumpkin balloon.

\section{Lobe Cutting Patterns}

A smooth, axi-symmetric surface that carries a uniform differential pressure $p$ purely with meridional stresses (i.e. the hoop stress is zero everywhere) is called an isotensoid. The shape of its meridian was first derived by G.I. Taylor ${ }^{1}$ using a direct equilibrium formulation and later from variational calculus in Refs. ${ }^{9,10}$ It will be called Taylor's curve in the following. Note that the pressure in the balloon will be assumed to be uniform; pressure variation effects have been analysed in Ref. ${ }^{11}$

Since a pumpkin balloon carries the differential pressure mainly through meridional stresses with the help of tendons, it can be shown that the profile of the tendons converges to Taylor's curve if the number of lobes, $n$, tends to infinity. Unfortunately, there exists no closed form solution of Taylor's curve in terms of elementary functions. Therefore we will express Taylor's curve with the help of an elliptic integral that can be solved numerically

$$
y(x)= \pm \int_{x}^{x_{0}} \frac{\zeta^{2}}{\sqrt{x_{0}^{4}-\zeta^{4}}} d \zeta
$$

where the $y$-axis goes through the apexes and $x_{0}$ is the radius at the equator.

Figure 4 shows a series of cross-sections of an inflated lobe; each cross-section is identified by the variable 
$\xi \in[-1,1]$, measured along the arc-length of the tendons. The cross-sections are close to circular-arcs and it can be shown that, on the assumption that the pressure is carried by the membrane directly towards the tendons, the cross-sections of the lobes converge to circular-arcs for $n \rightarrow \infty$.
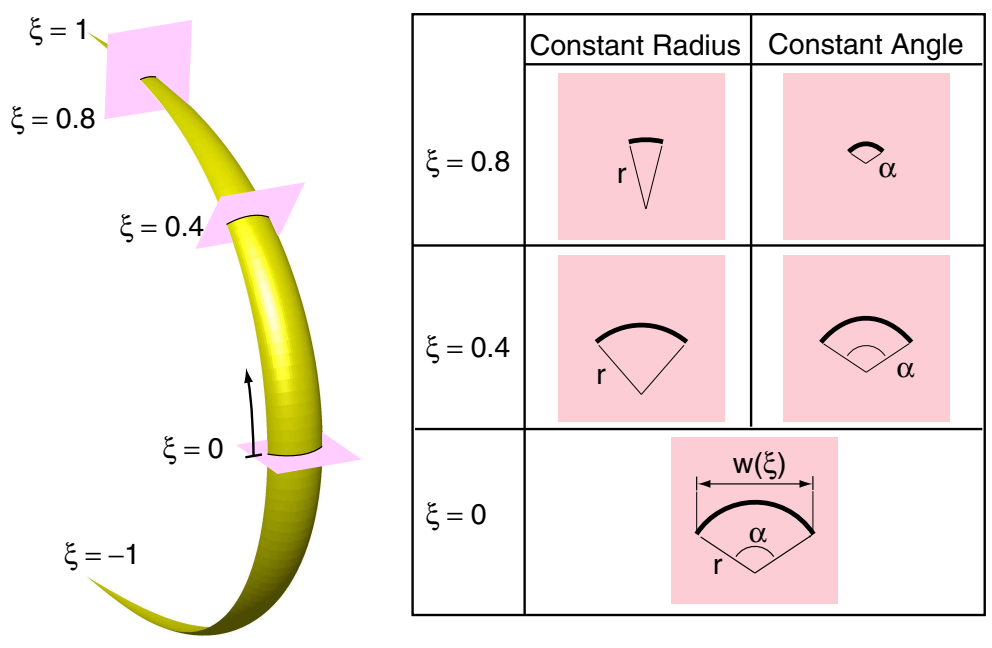

Figure 4. Cross-sections of an inflated lobe.

A brief outline of the argument is given in the following. The functional that governs the shape of the cross-section of a lobe, in a $x-y$ coordinate system with the start and end points of $x=-a$ and $x=a$, is

$$
F\left(y, y^{\prime}\right)=\int_{-a}^{a}\left(-r y-y^{2}+\lambda\left(\sqrt{1+y^{\prime 2}}-\frac{L}{2 a}\right)\right) d x .
$$

The curvature of the tendons at the point where the shape of the lobes is evaluated is $1 / r$ and the total arc-length of the lobes cross-section is $L$ which is introduced with the help of the Lagrangian multiplier $\lambda$. It can be seen that if $r$ becomes large, or $y$ small (which is the case if $n \rightarrow \infty$ ) then the functional provides the equation of a circular-arc.

To determine the cutting-pattern of the lobes we make the following two key assumptions

- The tendon profile is given by Taylor's curve;

- The cross-sections of the lobes are circular-arcs.

With these assumptions, it is possible to derive the cord-length, $w(\xi)$, i.e. the distance between two consecutive tendons, Figure 4, as a function of the tendon arc-length ${ }^{\mathrm{a}}$ by generating the cartesian coordinates of two identical sets of points lying on a Taylor curve and rotating one set about the axis of the balloon through an angle of $2 \pi / n$.

Next, two particular cutting-patterns for the lobes are considered, the so-called constant angle (CA) and constant radius $(\mathrm{CR})$ cutting-patterns. Note that neither cutting-pattern is in general optimal, however, these are the main cutting-patterns that have been considered so far for the ULDB balloons and hence are of great interest for this study. A cutting-pattern is defined by a function $w^{\prime}(\xi)$, obtained by increasing the cord length $w(\xi)$ by an amount $\Delta w(\xi)$. Figure 5 shows a cross-section of a lobe.

For a CA cutting-pattern $\Delta w(\xi)$ is chosen such that the resulting lobe width $w^{\prime}(\xi)$ is identical to the length of a circular-arc that subtends a constant angle, $\alpha_{C A}$, throughout the lobe. Therefore we get

$$
w^{\prime}(\xi)=\frac{w(\xi) \alpha_{C A}}{2 \sin \left(\alpha_{C A} / 2\right)}=\frac{y(\xi) \alpha_{C A} \sin (\pi / n)}{\sin \left(\alpha_{C A} / 2\right)} .
$$

aThe total arc-length of the tendons depends on the desired volume of the balloon, which can be estimated by computing the volume of a surface of revolution having Taylor's curve as the generator. This gives $V \approx 2.7458 r^{3}$ where $r$ is the radius of the balloon at the equator. 
For a CR cutting-pattern $\Delta w(\xi)$ is chosen such that the resulting lobe width $w^{\prime}(\xi)$ is identical to the length of a circular arc of constant radius $r_{C R}$. Hence in this case we have

$$
w^{\prime}(\xi)=2 r_{C R} \arcsin \left(\frac{w(\xi)}{2 r_{C R}}\right)=2 r_{C R} \arcsin \left(\frac{y(\xi) \sin (\pi / n)}{r_{C R}}\right) \text {. }
$$

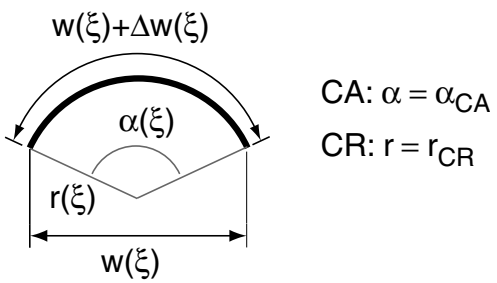

Figure 5. Lobe cross-sections for Constant Angle (CA) and Constant Radius (CR) cutting-patterns.

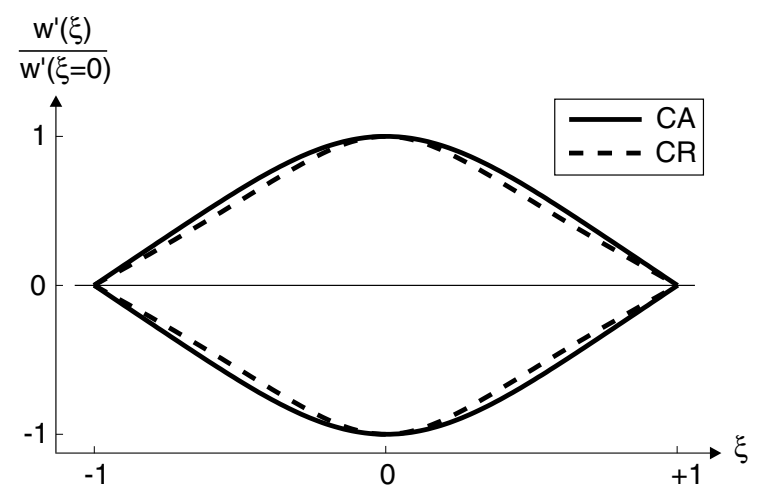

Figure 6. Cutting-patterns for balloons with identical subtended angle at the equator.

Figure 6 shows the resulting cutting-patterns for two balloons that have identical subtended angles and radii at the equator. It can be seen that the CA cutting-pattern has a larger area than the CR design. Note that the largest difference in width between the two patterns is around $\xi \pm 0.5$

\section{Numerical Modeling of Balloon Structure}

\section{A. Finite Elements}

The simulations presented in this paper are done with a newly developed, purpose made finite element program based on Matlab. ${ }^{12}$ For the discretisation of the membrane we use geometric non-linear three-node triangular membrane elements. The tendons are modeled with geometric non-linear two-node bar elements. In order to compute the stability of an inflated structure it is essential to take into account the load-following stiffness resulting from the pressure.

It should be noted that the global load following stiffness matrix of a balloon is symmetric whereas the load following stiffness matrix of a single lobe is asymmetric. This is because for any structure subjected to pressure forces the resulting global stiffness matrices are symmetric if the structure is closed or the boundary conditions fulfill certain criteria. ${ }^{13}$ In summary we can say that the load following stiffness matrix in a balloon forming a closed envelope is related to changes in the energy of the gas due to changes in the enclosed volume.

\section{B. Inflated Shape of a Lobe}

Since a pumpkin balloon consists of $n$ identical lobes, it is possible to simulate the inflation by only taking account of a single cutting-pattern whose boundaries can move in the designated symmetry planes of an 
arbitrary lobe. The inflation of a cutting-pattern is simulated in three stages, as follows

- Generation of discretised cutting-pattern;

- Moving boundary nodes to points lying on Taylor's curves in two meridional symmetry planes and applying differential pressure $p$;

- Releasing the cutting-pattern in its symmetry planes and computing final equilibrium.

The first stage can be split into two sub-steps. The first sub-step, shown in Figure 7, creates the cuttingpattern between the tendons. The second sub-step consists in discretising the cutting-pattern of the lobe, as shown in Figure 8(a). It should be noted that the mesh of the membrane has to be compatible along the boundaries with the mesh adopted for the tendons. Furthermore, it is best for the symmetry transformation that will be described later, that the chosen finite-element discretisation is symmetric with respect to the equatorial plane of the balloon and the mid-plane of the lobe ${ }^{\mathrm{b}}$.

The second stage of the inflation process moves the boundary nodes back to their original position on Taylor's curve - lying on the corresponding symmetry planes of the lobe - and simultaneously applies a differential pressure $p$ that acts on the membrane. The application of $p$ is necessary to avoid a singular stiffness matrix since the membrane elements do not have any bending stiffness. It should be noted that the tendons are stress- and strain-free after this stage since they are back on their original location. The resulting forces on the tendons due to the pressure load on the membrane are carried by additional forces that are introduced by the Lagrangian multiplier to enforce the prescribed displacements. Figure 8(b) shows a lobe after the second stage.

The third stage allows the tendons to move free within the symmetry planes in order to reach their equilibrium configuration while the shape of the lobe changes to a certain extent. These shape changes are rather small, since Taylor's curve tends to be the exact shape for the tendons of pumpkin balloons with $n=\infty$. An inflated lobe in equilibrium after the third stage is shown in Figure $8(\mathrm{c})$. The tendons from the second stage of the computation are also shown in the figure, coloured in red for clarity. It should be mentioned that the differences in this picture are far greater than those found in pumpkin balloons with a realistic number of lobes.

The discretisations of the cutting-patterns that are used throughout this paper are chosen such that they preserve the horizontal and vertical reflection symmetry of the cutting-patterns and can be defined by the number of elements along the tendons and the equator.

If we take, for example, a $10 \mathrm{~m}$ diameter balloon then it is obvious that the width of the cutting-patterns is a function of the number of lobes, $n$. Therefore it is necessary that the number of elements along the tendons and the equator be varied in order to preserve an acceptable aspect ratio. Table 1 provides details on the discretisations used and the resulting total number of elements and nodes. Hence, a balloon with $n=145$ has $(145 \times(1671-(134+1))+2) \times 3=668,166$ degrees of freedom (dofs). Therefore, real flight balloons with $n \approx 280$ have more than $10^{6}$ dofs.

\begin{tabular}{l|ccc}
$n$ & $16-48$ & $64-96$ & $112-145$ \\
\hline Elements along tendons & 100 & 116 & 134 \\
Elements at equator & 30 & 24 & 20 \\
\hline Number of elements & 3460 & 3216 & 3072 \\
Number of nodes & 1831 & 1725 & 1671
\end{tabular}

Table 1. Properties of discretised cutting patterns.

\section{End Fittings}

The tendons and the membrane of the lobes are terminated with the help of end-fittings at the top and bottom apexes. Furthermore, it is necessary to separate the tendons and the membrane a short distance

\footnotetext{
b It would also be possible to use a non-symmetric mesh since the symmetry transformation averages the corresponding nodal stiffnesses of symmetry related degrees of freedom. This is the reason why a symmetry adapted coordinate system can increase the accuracy of a computation.
} 


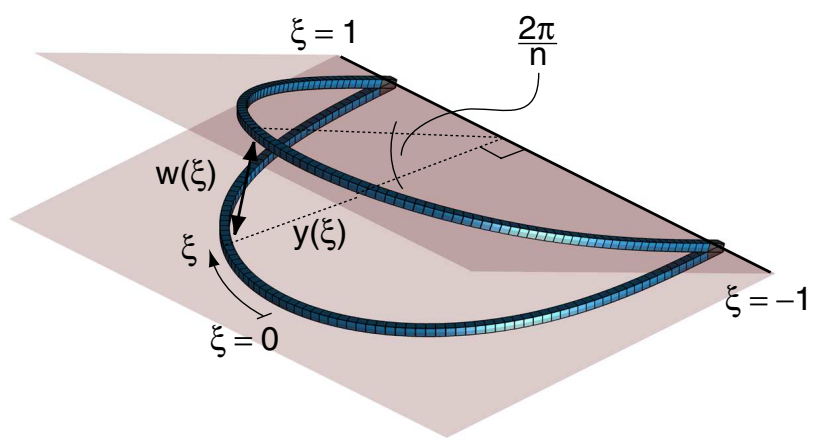

(a)

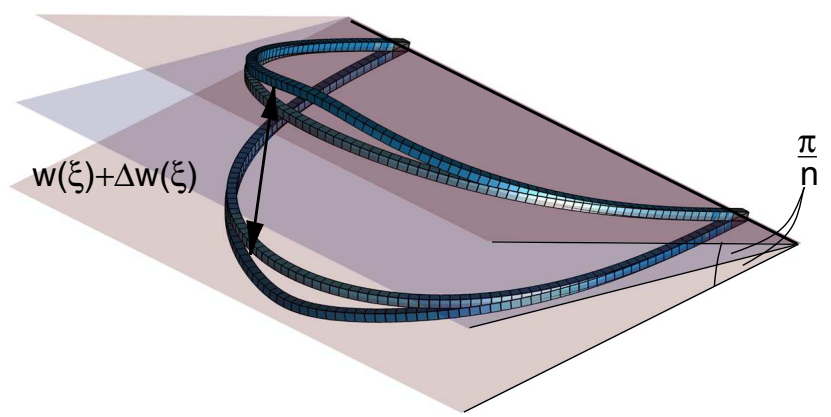

(b)

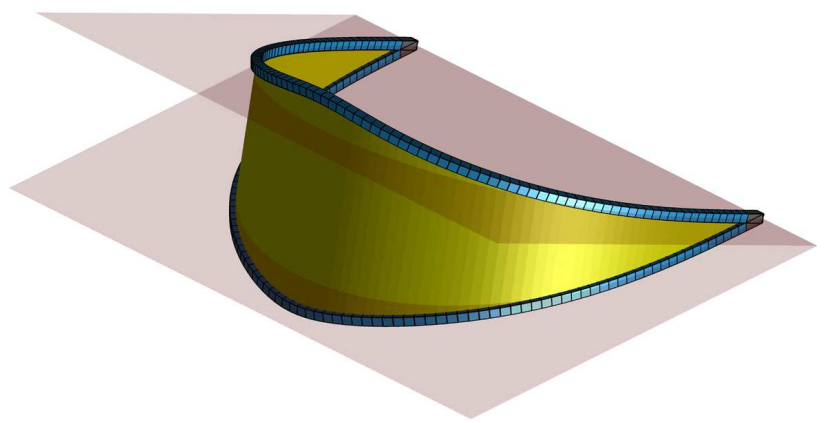

(c)

Figure 7. Definition of lobe geometry; (a) tendons of one lobe lying on Taylor's curve in their symmetry planes; (b) distance between tendons increased to $w^{\prime}(\xi)$; (c) resulting cutting-pattern. 

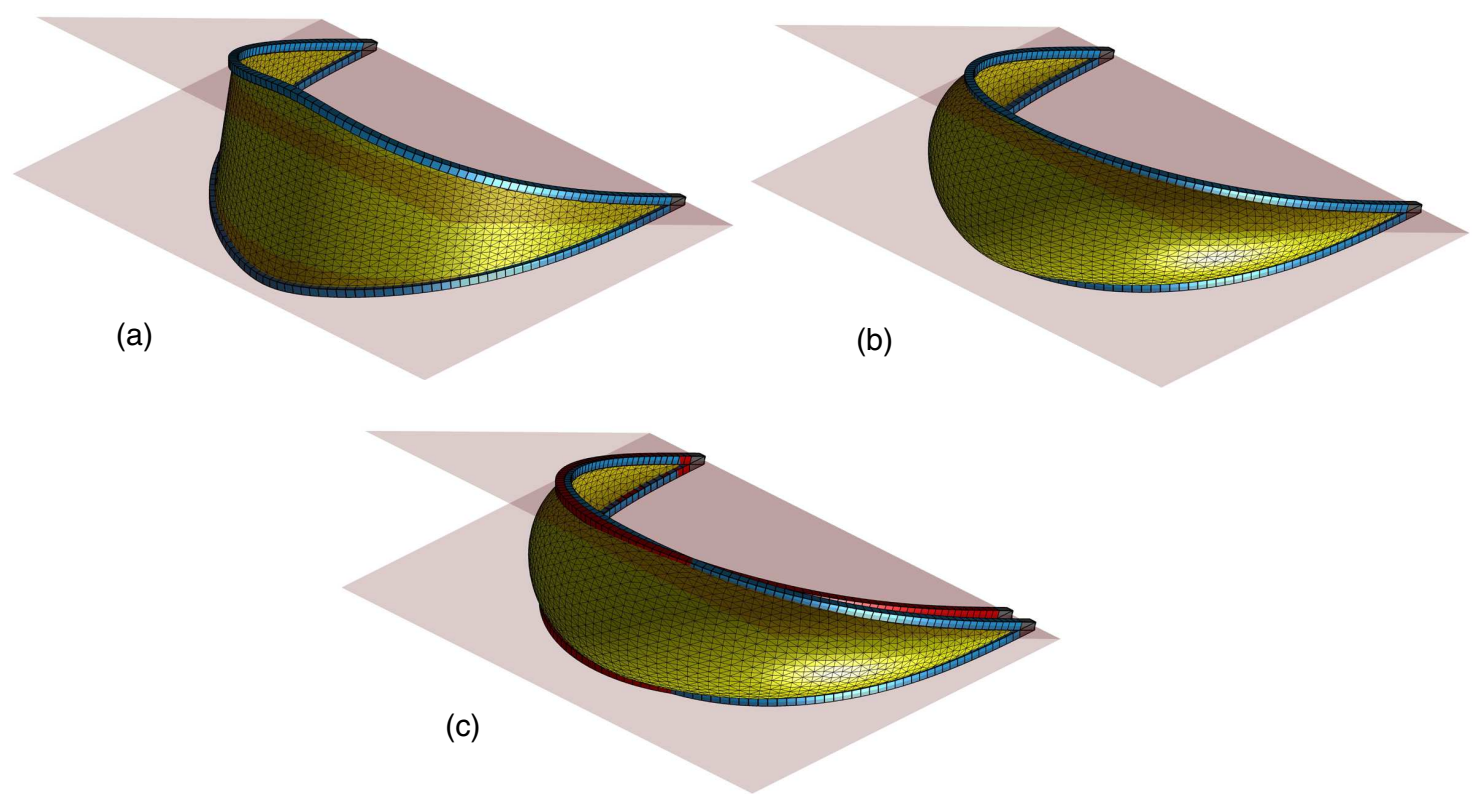

Figure 8. Procedure of cutting-pattern inflation; (a) Discretisation of cutting-pattern; (b) Application of differential pressure $p$ and movement of nodes back to symmetry planes; (c) Relaxing lobe in symmetry planes to reach final state of equilibrium.

before the end-fitting in order to clasp the membrane between the plates and to terminate the tendons at the ring.

In order to study the effects of the end-fittings on the stability of pumpkin balloons it is necessary to take them into account for the numerical simulations. It would be straightforward to model these stiff circular plates with the help of shell elements, but in order to keep the Matlab program simple, they were modelled with the already implemented bar elements.

\section{Lobe Superelements}

Superelements are groupings of finite elements that, upon assembly, may be considered as an individual element. They were invented by aerospace engineers in the early 1960s to improve the analysis efficiency of complex structures. Since each balloon consists of $n$ equal lobes, a superelement of one lobe is derived where all interior, non-boundary, nodes are eliminated with the help of static condensation. Although the size of the lobe stiffness matrix decreases considerably, the greatest advantage is that the symmetry transformation is simpler if there are only boundary nodes left. Therefore, it is possible to apply the transformation matrices, ${ }^{14}$ directly without any further modifications.

If the degrees of freedom are partitioned according to interior and boundary nodes, subscript $i$ and $b$ respectively, then it is possible to write

$$
\left(\begin{array}{ll}
\mathbf{K}_{b b} & \mathbf{K}_{b i} \\
\mathbf{K}_{i b} & \mathbf{K}_{i i}
\end{array}\right)\left(\begin{array}{l}
\mathbf{u}_{b} \\
\mathbf{u}_{i}
\end{array}\right)=\left(\begin{array}{c}
\mathbf{f}_{b} \\
\mathbf{f}_{i}
\end{array}\right)
$$

where $\mathbf{K}_{b b}$ and $\mathbf{K}_{i i}$ are submatrices that depend only on the subvectors of the boundary $\mathbf{u}_{b}$ and interior nodes $\mathbf{u}_{i}$. The second matrix equation from Equation 5 is

$$
\mathbf{K}_{i b} \mathbf{u}_{b}+\mathbf{K}_{i i} \mathbf{u}_{i}=\mathbf{f}_{i}
$$

which can be solved for the interior freedoms $u_{i}$ if $\mathbf{K}_{i i}$ is nonsingular

$$
\mathbf{u}_{i}=\mathbf{K}_{i i}^{-1}\left(\mathbf{f}_{i}-\mathbf{K}_{i b} \mathbf{u}_{b}\right) .
$$

Replacing the interior freedoms of the first equation from Equation 5 by inserting Equation 7 results in

$$
\widetilde{\mathbf{K}}_{b b} \mathbf{u}_{b}=\widetilde{\mathbf{f}}_{b}
$$


which are the condensed stiffness equations. In this equation $\widetilde{\mathbf{K}}_{b b}$ is

$$
\widetilde{\mathbf{K}}_{b b}=\mathbf{K}_{b b}-\mathbf{K}_{b i} \mathbf{K}_{i i}^{-1} \mathbf{K}_{i b}
$$

and $\widetilde{\mathbf{f}}_{b}$ can be derived as

$$
\widetilde{\mathbf{f}}_{b}=\mathbf{f}_{b}-\mathbf{K}_{b i} \mathbf{K}_{i i}^{-1} \mathbf{f}_{i} .
$$

In the following $\mathbf{K}_{\text {sup }}$ is used instead of $\widetilde{\mathbf{K}}_{b b}$ in order to emphasize that it is the stiffness matrix of a superelement. More details on superelements and references can be found in Felippa. ${ }^{15}$

Static condensation is in general only of interest for linear problems. This is evident if a geometricnonlinear simulation is considered where the superelement has to be recomputed if the displacements of the boundary nodes become too big. Therefore static condensation is applied in the following to a lobe that is already in equilibrium with a differential pressure $p$ where the condensed matrix serves as a basis for the computation of the eigenvalues and eigenvectors of a symmetry transformed global stiffness matrix. It is important to note that a superelement has to be constructed at every differential pressure $p$ for which the stability of a balloon is simulated.

Figure 9 shows the partitioning of the original stiffness matrix of a lobe. The condensed matrix $\mathbf{K}_{\text {sup }}$ is fully populated but its size is only that of the sub-matrix $\mathbf{K}_{b b}$.

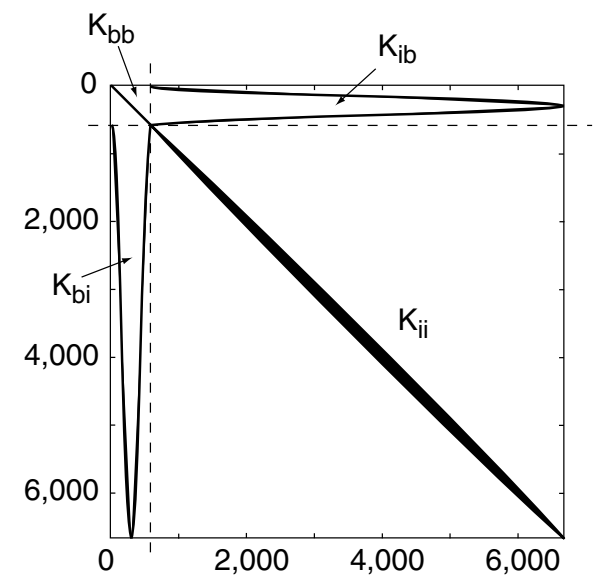

Figure 9. Partitioned stiffness matrix of a lobe.

\section{Block Diagonalised Stiffness Matrix}

Pumpkin balloons belong to the $D_{n h}$ symmetry group if a constant differential pressure $p$ is assumed and the influence of gravity is neglected. Since the error, introduced through these assumptions, is negligible it is shown in the following how to exploit the symmetry of pumpkin balloons for the computation of their stability.

A structure that belongs to a $D_{n h}$ symmetry group has a single $n$-fold rotation axis, one horizontal mirror plane, $n$-twofold axes perpendicular to the principal axis and $n$ vertical mirror planes. This is illustrated in Figure 10 with the help of a 16 lobe pumpkin balloon $\left(D_{16 h}\right)$. It is shown in Ref. ${ }^{14}$ that it is possible to split each frequency of the symmetry transformation into four decoupled blocks. However, it is not done here since the resulting block for each frequency is already small enough for practical purposes.

Let $\mathbf{T}_{i}$ be the $q \times r$ symmetry transformation matrix of a single frequency $i$ whose bounds are defined with the help of the number of lobes $n$

$$
i \in\left[0, \frac{n-n \bmod 2}{2}\right] .
$$

The number of rows $q$ depends on the number of lobes $n$ and on the number of elements $e_{t}$ that are used for discretising one tendon such that

$$
q=3 n\left(e_{t}+3\right) .
$$




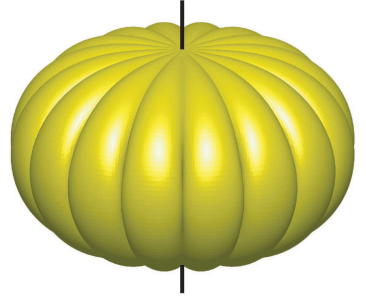

(a)

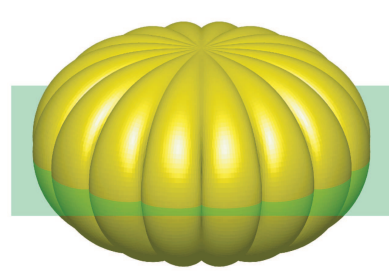

(b)

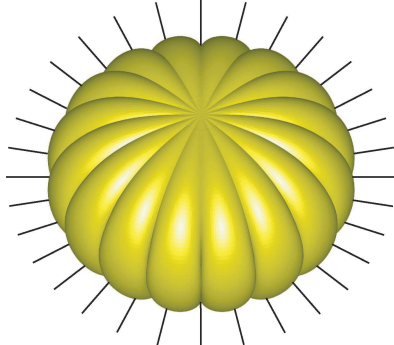

(c)

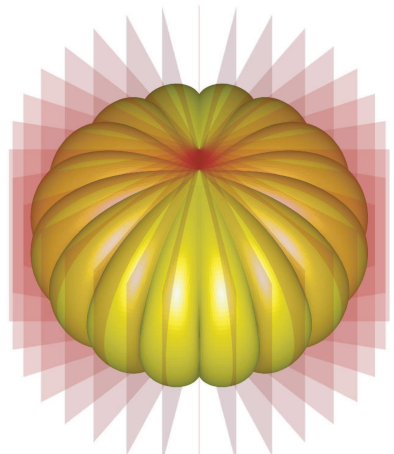

(d)

Figure 10. Symmetry planes and rotation axes of pumpkin balloon with 16 lobes; (a) $n$-fold rotation axis; (b) horizontal mirror plane; (c) $n$-twofold axes perpendicular to principal axis; (d) $n$ vertical mirror planes.

It should be noted that $q$ contains the two additional nodes from the support structure of the end-fittings. The number of column vectors $r$ is

$$
r=n_{e}+n_{t}+n_{p}
$$

where $n_{e}, n_{t}$ and $n_{p}$ are the numbers of the equatorial, tropical and polar orbits of $i$. With the help of $R_{\frac{2 \pi}{n}}$, a rotation matrix for the $n$-fold rotation axis, it is possible to write for the stiffness matrix block $\mathbf{K}_{i}$

$$
\mathbf{K}_{i}=\sum_{j=0}^{n-1} \mathbf{T}_{i}^{T}\left(\left(R_{\frac{2 \pi}{n}}^{j}\right)^{T} \mathbf{K}_{\text {sup }} R_{\frac{2 \pi}{n}}^{j}\right) \mathbf{T}_{i} .
$$

Theoretically it would be best to create the stiffness matrix of a complete balloon in a cartesian coordinate system prior to the symmetry transformation. This would considerably reduce the total number of matrix operations that are necessary during the simulations, since the transformation from a cartesian into a symmetry adapted coordinate system could be done in one step. Unfortunately it is not possible to do this for balloons with $n>16$ since the available computational capacity is limited. As the number of lobes of ULDB balloons is in general divisable by 16 it is advisable to create a stiffness matrix $K_{\text {sup }}$ that contains a patch of 16 lobes such that the total number of symmetry transformations of $K_{\text {sup* }}$ with $\mathbf{T}_{i}$ reduces to $\frac{n+15(n \bmod 16)}{16}$

$$
\mathbf{K}_{\text {sup }}=\sum_{j=0}^{16}\left(R_{\frac{2 \pi}{n}}^{j}\right)^{T} \mathbf{K}_{\text {sup }} R_{\frac{2 \pi}{n}}^{j}
$$

Therefore for the final assembly procedure used in the Matlab implementation is given by

$$
\begin{aligned}
\mathbf{K}_{i}= & \sum_{j=0}^{\frac{n-n \bmod 16}{16}-1} \mathbf{T}_{i}^{T}\left(\left(R_{\frac{32 \pi}{n}}^{j}\right)^{T} \mathbf{K}_{\text {sup }} R_{\frac{32 \pi}{n}}^{j}\right) \mathbf{T}_{i} \\
& +\sum_{j=n-n \bmod 16}^{n-1} \mathbf{T}_{i}^{T}\left(\left(R_{\frac{2 \pi}{n}}^{j}\right)^{T} \mathbf{K}_{\text {sup }} R_{\frac{2 \pi}{n}}^{j}\right) \mathbf{T}_{i} .
\end{aligned}
$$

It is shown later that we are in general only interested in eigenvalues and eigenvectors that correspond to frequencies $i \in[1,4]$ so that it is possible to neglect most of the blocks of $\mathbf{K}_{i}$. Since the number of blocks that are of interest is very small the computational complexity reduces extremely so that even huge problems can be solved on standard personal computers in only a couple of minutes.

\section{Properties of Test Balloons}

The geometric and material properties of a series of experimental balloons that have been investigated as part of the ULDB development will serve in the following as a basis for our computations. They are as follows. 


\section{- Geometric Properties}

- Number of lobes: $16-145$

- Total height: $5.99 \mathrm{~m}$

- Radius of tendons at equator: $5.00 \mathrm{~m}$

- Total length of tendons: $13.11 \mathrm{~m}$

- Cutting pattern: $C A$ and $C R$ with $118^{\circ}$ subtended angle at equator

- Radius of end-fittings: $0.29 \mathrm{~m}$

\section{- Material Properties ${ }^{\mathrm{c}}$}

- Young's modulus of membrane: $138.70 \mathrm{~N} / \mathrm{mm}^{2}$

- Poisson's ratio of membrane: 0.566

- Thickness of membrane: $0.038 \mathrm{~mm}$

- Axial stiffness of tendons: $170 \times 10^{3} N$.

In Table 2 we introduce a notation that is used in the following to describe the geometric properties of balloons that are studied in the following.

\begin{tabular}{ccccc}
$\mathbf{1 4 5}$ & $\mathbf{C R}$ & $\mathbf{1 1 8}$ & $\mathbf{1 0}$ & $\mathbf{2 9 0}$ \\
\hline $\begin{array}{c}\text { Number } \\
\text { of } \\
\text { lobes }\end{array}$ & $\begin{array}{c}\text { Constant radius } \\
\text { cutting } \\
\text { pattern }\end{array}$ & $\begin{array}{c}\text { Subtended } \\
\text { angle of } 118^{\circ}\end{array}$ & $\begin{array}{c}\text { Diameter } \\
\text { at equator balloon }\end{array}$ & $\begin{array}{c}\text { Diameter of } \\
\text { at equator is } 10 \mathrm{~m} \text {-fittings } \\
\text { is } 290 \mathrm{~mm}\end{array}$
\end{tabular}

Table 2. Notation for geometric properties pumpkin balloons. The balloon 145CR118-10-290 is considered as an example.

\section{Stress Distribution}

A drawback of using developable cutting patterns is that the membrane experiences high strains due to the incompatibility between the initial developable surface and the doubly curved shape required for the lobe to carry the pressure efficiently. These undesirable strains occur at very small pressures, and have their maximum at the centre of the lobes where the Gaussian curvature reaches its largest value. This can be understood by looking at the plane strain compatibility equation, ${ }^{16}$ from membrane theory

$$
K=\frac{\partial^{2} \gamma_{x y}}{\partial x \partial y}-\frac{\partial^{2} \varepsilon_{x}}{\partial y^{2}}-\frac{\partial \varepsilon_{y}}{\partial x^{2}}
$$

which shows that a non-zero Gaussian curvature $K$ can only be obtained from a developable (flat) surface if there are strains in the membrane that satisfy Equation 17. Furthermore, also for small differential pressures $p$ there are wrinkles parallel to the tendons. These wrinkles disappear once the strains due to $p$ are large enough to create a doubly curved surface.

Simulating the inflation of a single lobe shows that the principal stresses are aligned with the meridional and hoop directions; the maximum stresses occur at the equator. The variation of these maximum principal stresses with pressure is plotted in Figure 11.

The first thing to note is that both stress components are clearly non-zero. The plot also shows that the variation of the hoop stress is linear and that of the meridional stress approximately bilinear. For small differential pressures the meridional stress increases at a much higher rate than the hoop stress. Because initially the lobe is not allowed to bulge out, its curvature in the hoop direction is very small and so most of the pressure load has to be carried meridionally. However, because the meridional curvature is relatively small, high stresses are induced even by relatively small pressure increases. Smaller hoop stresses gradually develop in the lobe and at a pressure of around $380 \mathrm{~Pa}$ the lobe is fully in tension.

These large meridional stresses are accompanied by large meridional strains, which allow the lobe to form the required bulge. The formation of the bulge can be analysed on Figure 12, which plots the deflection $d$

\footnotetext{
${ }^{\mathrm{c}}$ The membrane is a composite material and consists of a high-tenacity polyester fabric, a polyester film that serves as the main barrier to the helium and a polyethylene film that contains the gas and provides extra durability. The tendons are made out of PBO (poly(p-phenylene-2,6-benzobisoxazole)).
} 


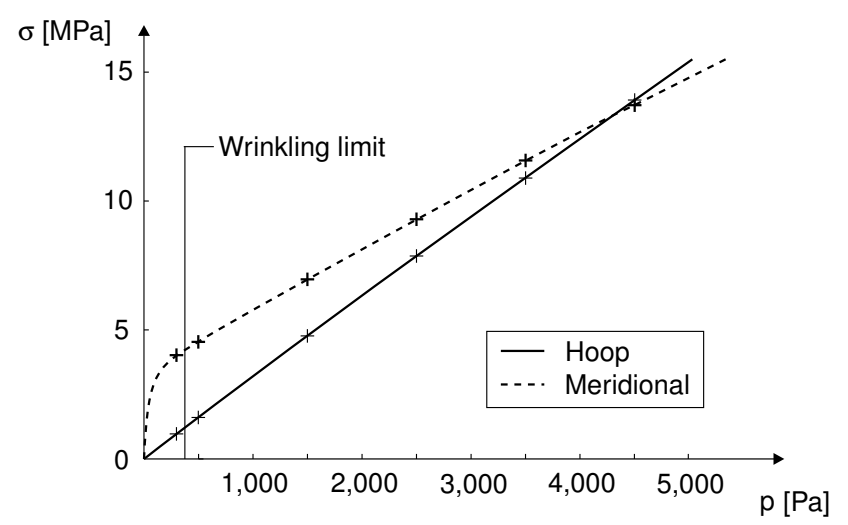

Figure 11. Maximum hoop and meridional stresses as a function of $p$ for a 145CR118-10-290 balloon.

at the centre of the lobe vs. the pressure $p$. Note that at around $800 \mathrm{~Pa} d$ reaches the value corresponding to the cutting pattern design.

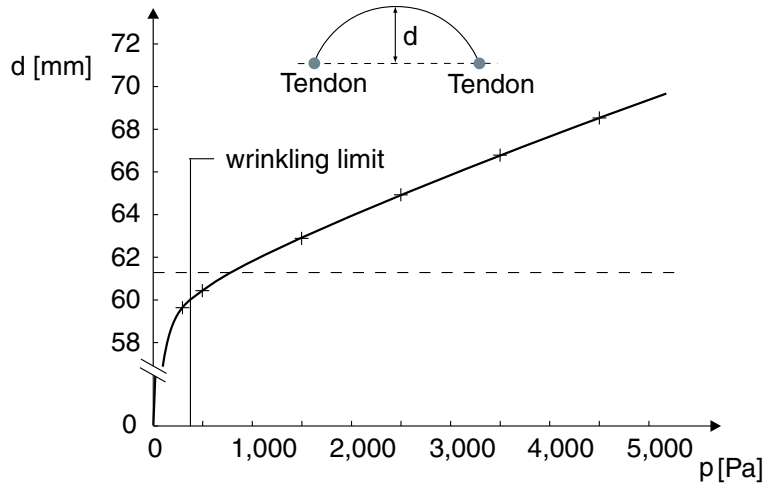

Figure 12. Deflection $d$ at centre of lobes of a 145CR118-10-290 balloon as a function of $p$.

Once the wrinkles have disappeared, most of the additional pressure increase is carried by the hoop stress in the lobe. However, the meridional stress does not remain constant. Instead, it continues to increase linearly but now at a rate of about two thirds of the hoop stress. This is largely because the lobe is not allowed to develop meridional strain due to the constraint imposed by the stiff tendons. Setting the meridional strain equal to zero gives $\sigma_{m}=\nu \sigma_{h}=0.566 \sigma_{h}$; the additional increase is a direct effect of the pressure load.

Figure 13 shows contour plots of the first and second principal stresses of a 145CR118-10-290 and 145CA118-10-290 lobe for $p=500,1500,2500,3500$ and $4500 \mathrm{~Pa}$. Note that only one quarter of each lobe is shown for each pressure and balloon design. Also note that the first (major) principal stress is in the hoop or meridional direction depending on the balloon design and pressure applied. An analysis of the forces in the tendons shows that they are proportional to the differential pressure $p$ and nearly uniform over their whole length.

As the maximum and minimum principal stresses are nearly identical for CA and CR designs, as the two designs are identical at the equator, the material utilisation of a CA design is worse as there are lower average stresses in the membrane.

An important question in the analysis of the stability of pumpkin balloons is if one needs to worry about the effect of the wrinkles in the membrane. It has been shown in this section that, due to the low Young's modulus of the membrane, there are no wrinkles for differential pressures $\gtrsim 380 \mathrm{~Pa}$, as the meridional strains in the membrane are large enough to generate a doubly curved surface. Therefore, we can conclude that wrinkles are unimportant for studying the stability of currently used super-pressure pumpkin balloons since 


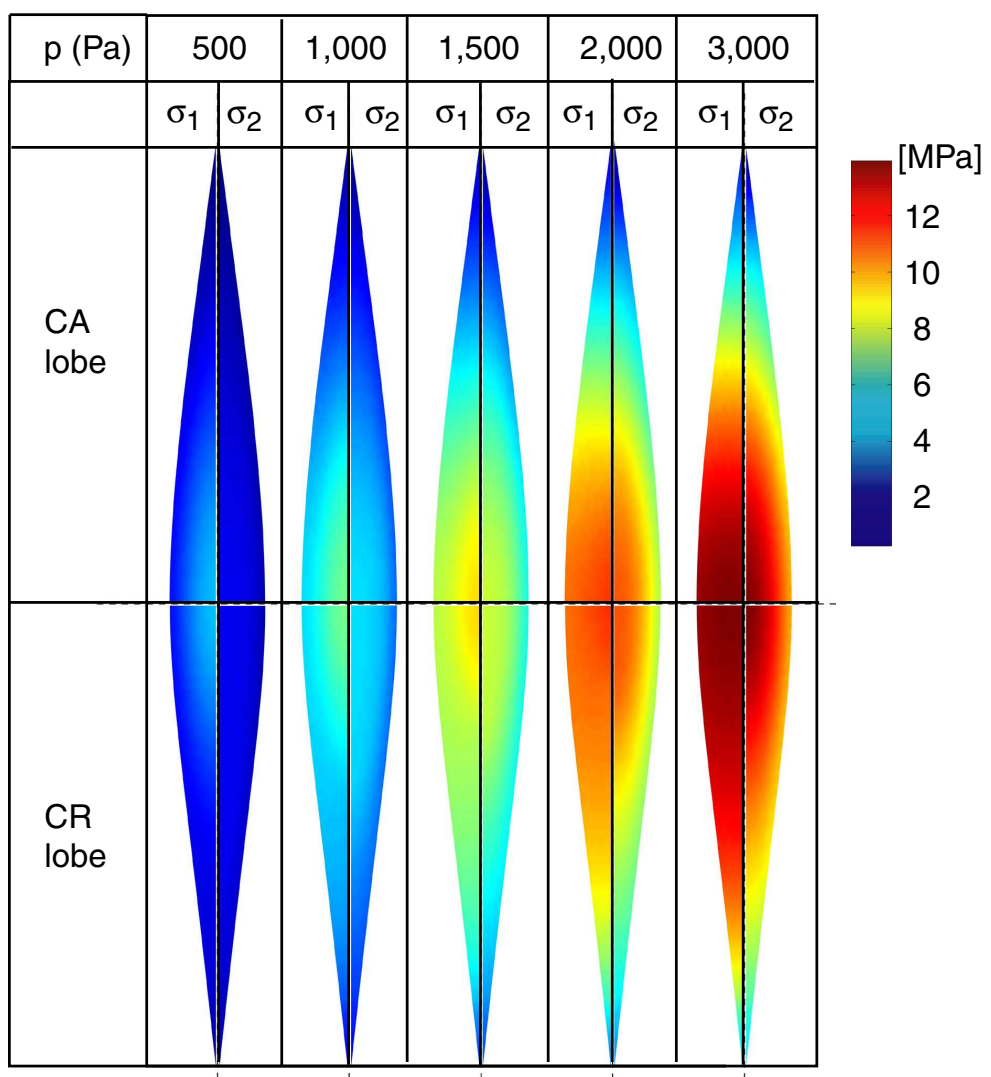

Figure 13. First and second principal stresses for cutting-patterns of 145CR118-10-290 and 145CA118-10-290 balloons (width of cutting-patterns is five times magnified). 
the operational differential pressure is much higher.

\section{Buckling of Balloons with 145 Lobes}

\section{A. Critical Pressure}

This section presents results of the stability simulation of pumpkin balloons with 145 lobes. In total, three different designs are considered, namely 145CA118-10-290, 145CR118-10-0 and 145CR118-10-290.

To get a meaningful measure for the stability of a balloon, that is independent of the mesh used, the eigenvalues are converted to deformation energy values, i.e. the work required to deform the balloon into the shape of a chosen eigenvector with a maximum nodal displacement of $1 \mathrm{~mm}$.

Figure 14 shows the two modes that turn out to be of particular importance for the stability simulations of the different designs. These modes are referred to as $i$ up $i$ down in the following, where $i$ is the wave number of the mode.

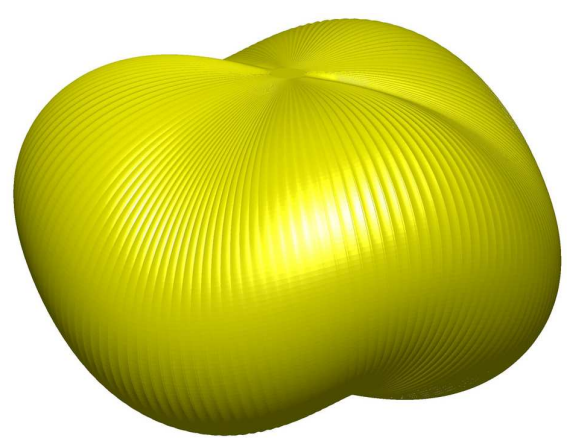

(a)

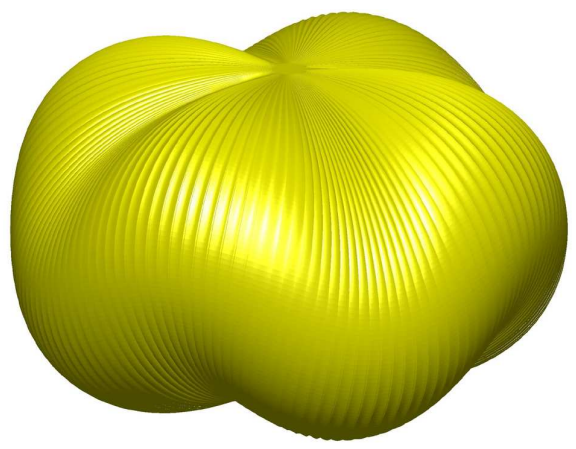

(b)

Figure 14. Modes of a 145CR118-10-290 balloon; (a) 3up3down; (b) 4up4down.

A plot of the variation of the deformation energy of a 145CA118-10-290 balloon with the pressure $p$ is shown in Figure 15. It can be seen that the balloon is unstable even for very small pressures; the first negative eigenvalue corresponds to a 3up3down mode. Therefore, inflating this balloon into the desired shape is impossible. Experimental results confirm these simulations and show that the final equilibrium shape belongs, like the 3up3down mode, to the $C_{3 v}$ symmetry group. Figure 16 shows the equilibrium shape of a 145CA118-10-290 balloon after inflation. It should be noted that the other two clefts cannot be seen in this picture.

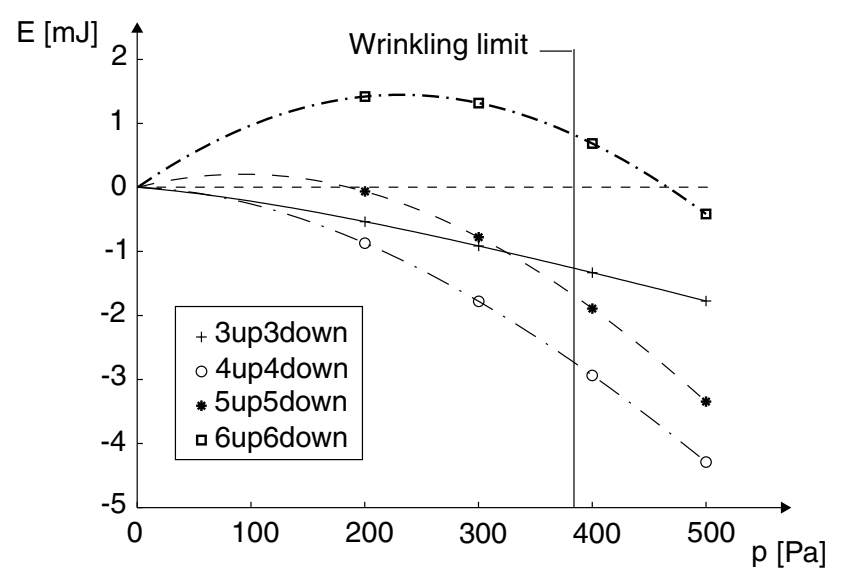

Figure 15. Deformation energy of four different eigenvectors versus pressure of 145CA118-10-290 balloon. 


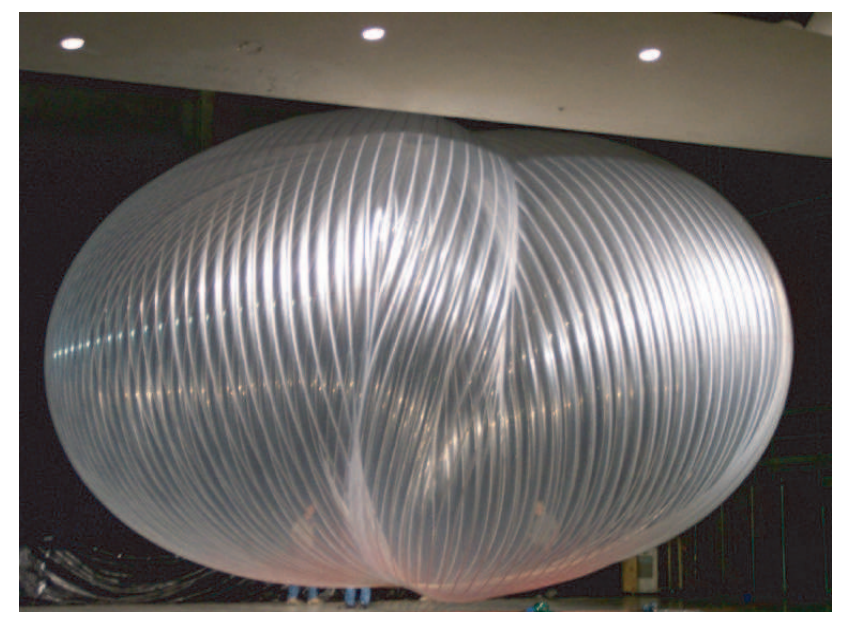

Figure 16. Inflated 145CA118-10-290 balloon with three clefts (courtesy of NASA).

The same study has been carried out for a CR balloon with similar characteristics, but initially without the end fittings. Figure 17 shows a plot of the variation of the deformation energy of a 145CR118-10-0 balloon with pressure. It can be seen that for small differential pressures all eigenvalues are positive and the initial tangent of the eigenvalues increases with the wave number $i$. Therefore a balloon with this design would inflate into the correct shape. At a pressure of $p \approx 3,040 \mathrm{~Pa}$ the eigenvalue which is associated with a 4up4down mode becomes zero and the balloon buckles.

Next the same balloon, but with end-fittings, was studied. This is to determine the influence of the end fittings on the stability of pumpkin balloons. The variation of the deformation energy of a 145CR118-10-290 balloon with pressure is shown in Figure 18. As in the case of the 145CR118-10-0 balloon, the critical buckling mode is 4up4down. Due to the end-fittings the critical differential pressure increases to $p \approx 3,500 \mathrm{~Pa}$ which is an increase of $15 \%$ compared to a balloon without end-fittings.

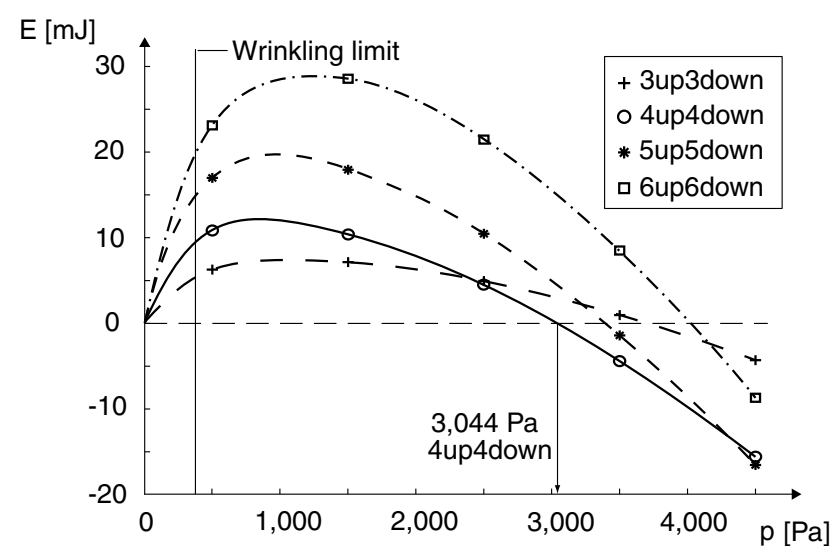

Figure 17. Stability of 145CR118-10-0 balloon (without end-fittings).

If the results for the stability of 145CA118-10-290 and 145CR118-10-290 balloons are compared, it becomes clear that the different cutting-patterns have a significant impact on the stability. This result is even more striking if it is taken into account that a CA cutting-pattern has comparable maximum stresses to a CR design for the same pressure. Therefore, it can be concluded that CA designs are not of practical interest. An implication of this result is that the accuracy with which the lobes are manufactured is crucial to reach the simulated buckling pressure of a perfect pumpkin balloon.

Finally, Figure 19 shows the critical pressures of a 145CR118-10-290 balloon for different frequencies $i$. It can be seen that the curve is convex and the critical mode is 4up4down. Hence, the critical mode $i$ is 


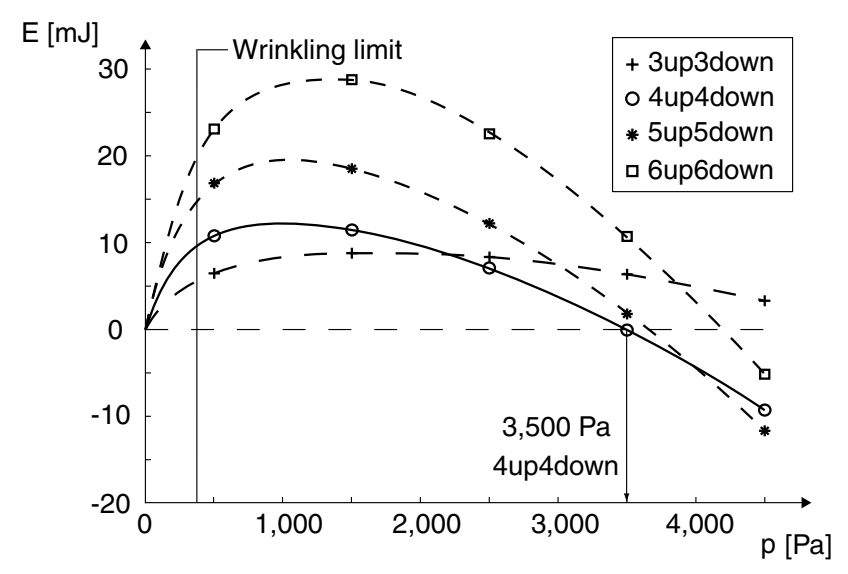

Figure 18. Stability of 145CR118-10-290 balloon (with end-fittings).

found if $i-1$ and $i+1$ have larger buckling pressures.

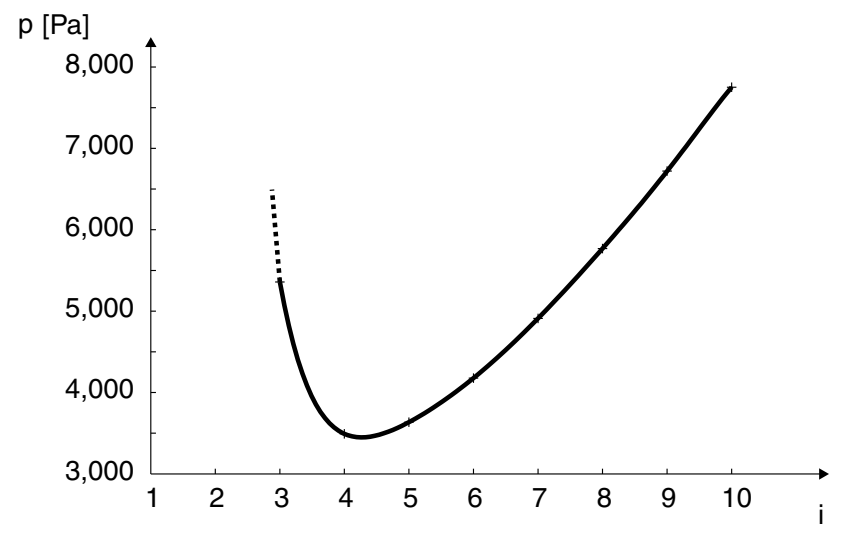

Figure 19. Buckling pressures of 145CR118-10-290 balloon for different frequencies $i$.

\section{B. Sensitivity Analysis}

Real balloons are constructed from materials that have properties which vary according to probability distributions. Therefore it is of interest to know how changes in the material parameters may affect the buckling pressure $p_{\text {crit }}$. Furthermore, it is of interest to estimate the quality of the numerical results obtained in the present study, by computing the sensitivity of $p_{\text {crit }}$ to a change in mesh density.

Figure 20 shows the sensitivities of $p_{\text {crit }}$ of a 145CR118-10-290 balloon to the Young's modulus $E_{m}$ and Poisson's ratio $\nu$ of the membrane as well as the stiffness of the tendons $E A_{t}$ and the lobe discretisation.

It becomes obvious from Figure 20(a) that the Young's modulus of the membrane has, compared with the other material parameters, the largest influence on the buckling pressure. Reducing $E_{m}$ by $10 \%$ results in a decrease of the buckling pressure by $14 \%$. This is mainly due to the fact that a smaller Young's modulus leads to larger strains in the cutting-pattern for the same differential pressures $p$. Therefore the subtended angle of the lobes increases and the buckling pressure decreases.

A similar effect can be observed if the Poisson's ratio $\nu$ of the membrane is decreased, Figure 20(b). Since the strains in meridional and hoop direction are coupled by the Poisson's ratio a decrease of $\nu$ increases the strains in the hoop direction. Therefore the subtended angle becomes larger and the buckling pressure smaller. Decreasing $\nu$ by $10 \%$ reduces the buckling pressure by $9 \%$.

The sensitivity of the tendon stiffness $E A_{t}$ is an exception since a decrease of $E A_{t}$ by $10 \%$ increases the buckling pressure by $3 \%$, Figure $20(\mathrm{~d})$. This is due to an elongation of the tendons which increases the width 


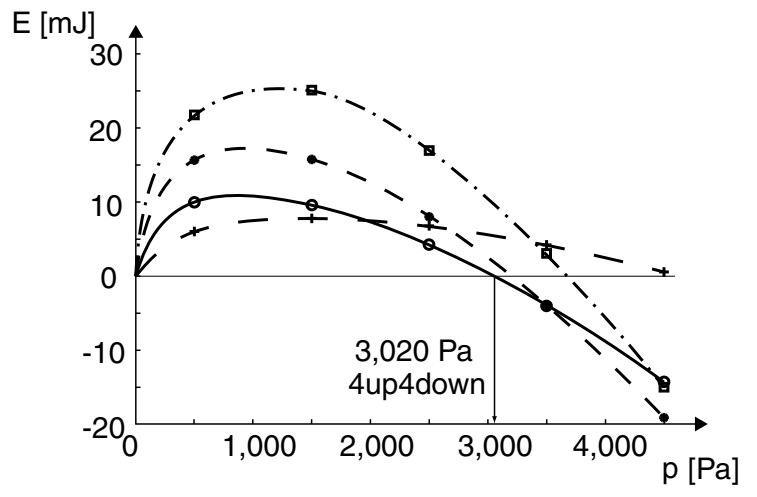

(a) $-10 \% E_{m} \rightarrow-14 \% p_{\text {crit }}$

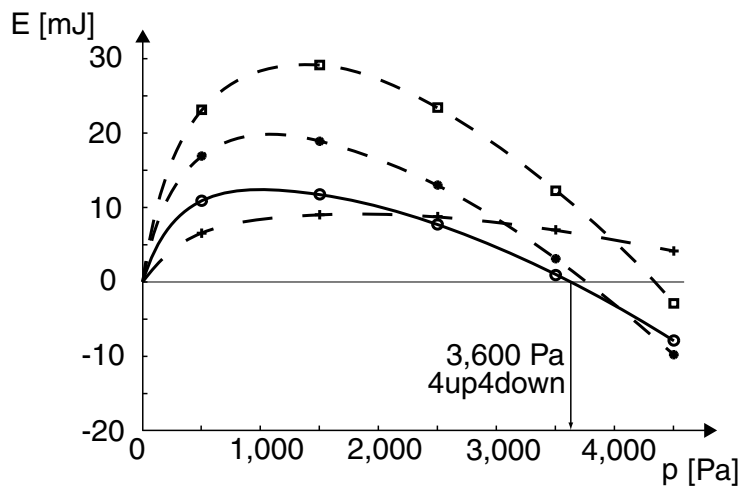

(c) $-10 \% E A_{t} \rightarrow+3 \% p_{\text {crit }}$

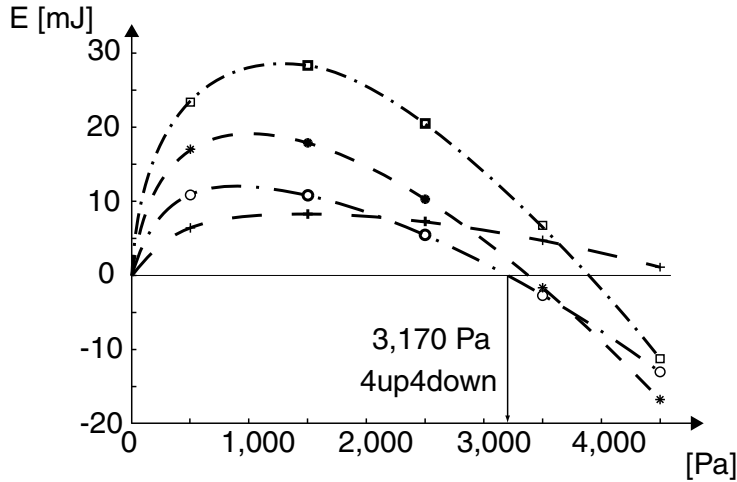

(b) $-10 \% \nu \rightarrow-9 \% p_{\text {crit }}$

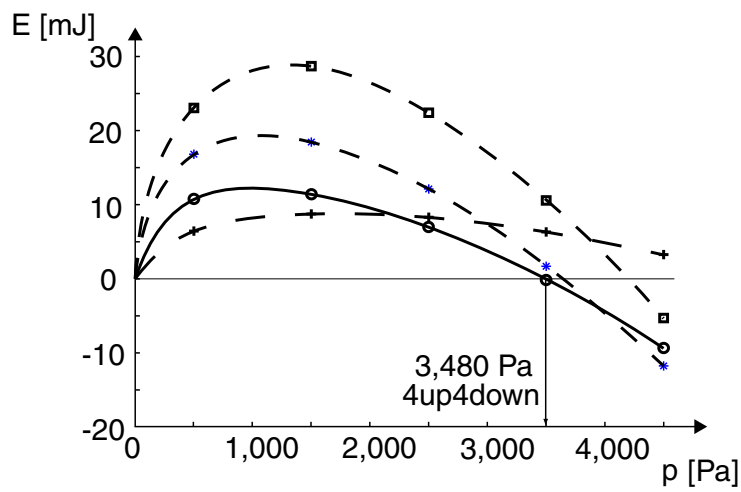

(d) $-20 \%$ elements at equator $\rightarrow-0.2 \% p_{\text {crit }}$

Figure 20. Sensitivities of deformation energy, for 145CR118-10-290 balloon, to material parameters and mesh density. See Figure 18 for legend. 
of the lobes. Therefore the subtended angle decreases and the stability of the balloon increases. However, this increase in stability comes with an increase in the stress levels, due to the smaller curvature in hoop direction and the additional elongation of the cutting-pattern in meridional direction.

Finally, Figure 20(d) shows the sensitivity of the buckling pressure on the mesh density. Reducing the number of finite elements at the equator by $20 \%$ and linearly to $0 \%$ at the apexes, results in a decrease of the buckling pressure by $0.2 \%$. This leads to the conclusion that the discretisation used in the present study is fine enough to give acceptable results.

\section{Balloons with Any Number of Lobes}

\section{A. Critical Pressure}

To decrease the membrane stresses in a pumpkin balloon that has to resists a given pressure, it is necessary to increase the curvature of the membrane in the hoop direction. This can be done by increasing either the number of lobes or the subtended angle of the lobes. However, both options have the disadvantage that they decrease the buckling pressure. In the following the dependence of the buckling pressure on the number of lobes $n$ is investigated.

Figure 21 shows a plot of the buckling pressure of a $n$ CA118-10-290 balloon and Figure 22 of a $n$ CR11810-290 balloon. It can be seen that the stability of both balloons increases exponentially with decreasing number of lobes. The buckling pressures of the $n$ CA118-10-290 balloons are relatively low for a large range of $n$ where the critical mode changes from 4up4down to 3up3down for $n \gtrsim 96$. It should be mentioned that the lobe has wrinkles for $p \lesssim 380 P a$. Since these wrinkles are not modeled in the presented simulations (and so the lobes are allowed to carry compressive stresses) the buckling pressures will be overestimated.

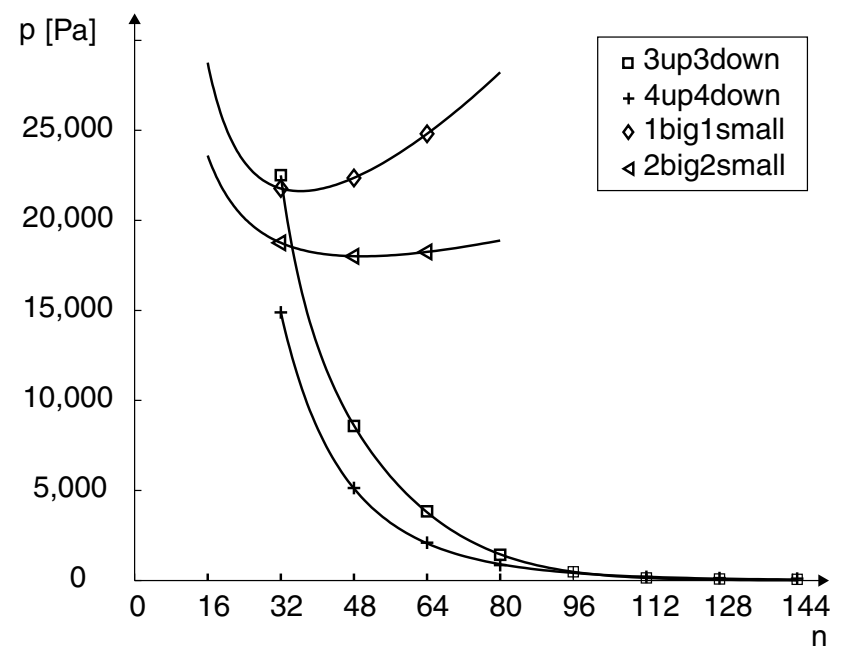

Figure 21. Buckling pressures of $n$ CA118-10-290 pumpkin balloons as a function of lobe number $n$.

A remedy for this would be the application of a finite element that simulates wrinkled membranes. However, since pumpkin balloons that buckle in such a low pressure region are not of practical interest, no further investigations of wrinkled lobes are attempted.

The buckling pressures of the $n$ CR118-10-290 balloons are much larger than those of the $n$ CA118-10-290 balloons. Furthermore there is no crossing of critical modes so that the balloons generally buckle into a 4up4down mode. Generally, because for $n$ CA118-10-290 and $n$ CR118-10-290 designs there is a new kind of critical mode for small numbers of $n$ and very large differential pressures $p$. They preserve the horizontal symmetry - in contradiction to the $i$ upidown modes - and have regions where the lobes are contracted or expanded as shown in Figure 23 for the case of a 32CR118-10-290 balloon. In the following these modes are denoted as $i$ bigismall due to the presence of contraction and expansion zones. Actually these modes are only of academic interest since they are only relevant for balloons with a small number of lobes at unrealistically high pressures.

As already mentioned the buckling pressure increases exponentially with decreasing number of lobes. 


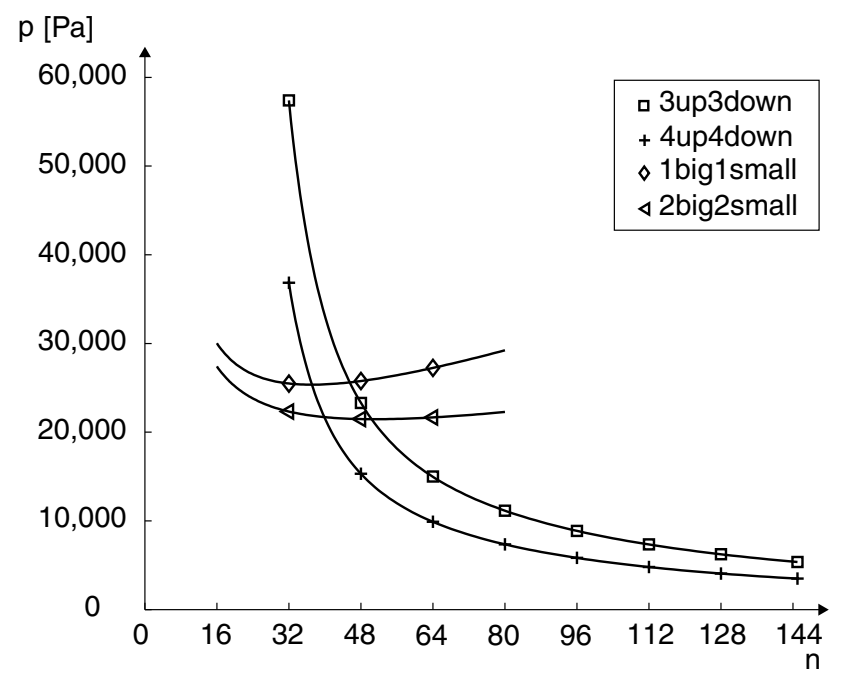

Figure 22. Buckling pressures of $n$ CR118-10-290 pumpkin balloons as a function of lobe number $n$.

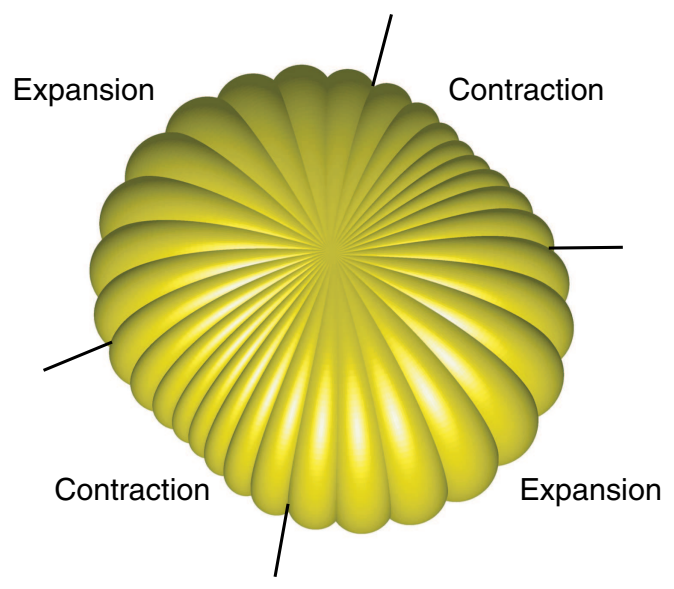

Figure 23. 2big2small mode of 32CR118-10-290 balloon. 
This becomes obvious if Figure 21 is re-plotted using log-axes. Figures 24(a) and 24(b) show this type of plot respectively for $n$ CA118-10-290 and $n$ CR118-10-290 balloons.

The variation is practically linear for the CR balloons. The slight deviation from a straight line for $n \rightarrow 1$ is due to stiffening effects as the overall shape of the balloons changes from Taylor's curve towards a sphere. The stability curve of the CA designs does not show such a pleasing linear behaviour. This is probably due to the influence of the shape changes of the wrinkled lobes for buckling pressures $\lesssim 380 P a$ and the already mentioned effects for small $n$ 's.

In the following only CR designs are investigated. Since the relationship between the buckling pressure $p_{\text {crit }}$ and the number of lobes $n$ is linear in a $\log -\log$ plot, their relationship is a power law

$$
p_{\text {crit }}=a n^{k}
$$

where $a$ is the constant of proportionality and $k$ the exponent of the power law. These coefficients are functions of the cutting-pattern and the material parameters.

If the buckling curve of $n$ CR118-10-290 balloons on a log-log plot, Figure 24(b), is linearly interpolated for $n \geq 64$ then $a=6.2917$ and $k=-1.2733$.

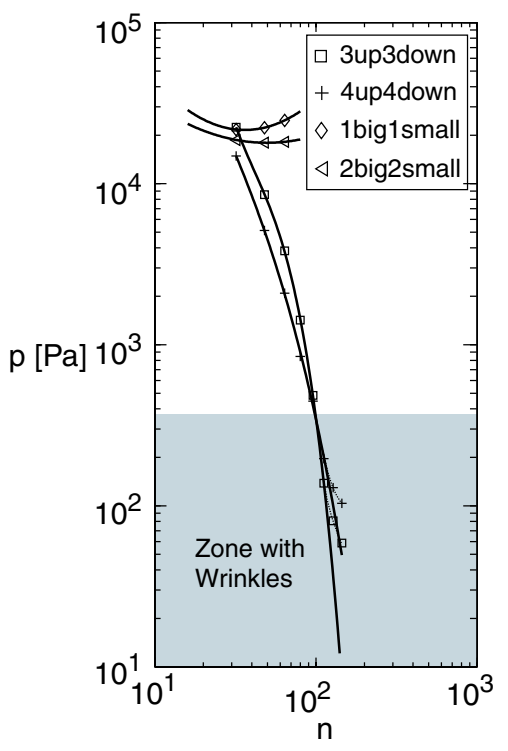

(a) Stability of $n$ CA118-10-290

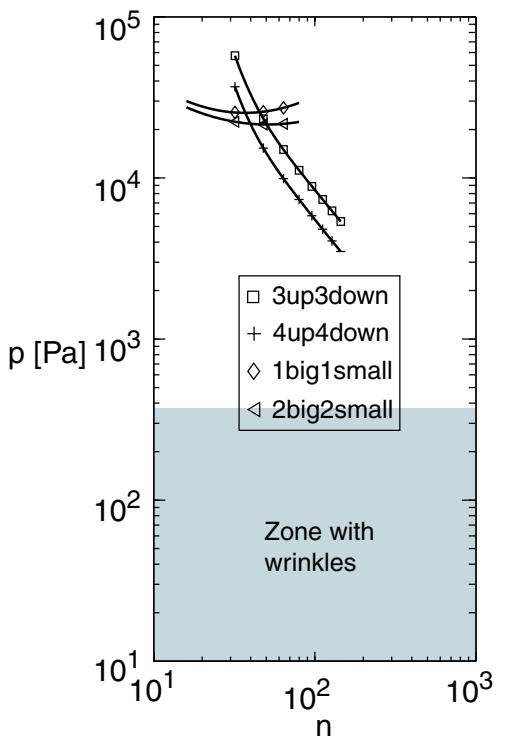

(b) Stability of $n$ CR118-10-290

Figure 24. Buckling pressures of balloons as a function of lobe number $n$ in a log-log plot.

\section{B. Sensitivity Analysis}

This section contains a study of the sensitivities of $n$ CR118-10-290 balloons on the material parameters, Figure 25. As in the case of the 145CR118-10-290 balloon, a decrease of the Young's modulus $E_{m}$ or Poisson's ratio $\nu$ of the membrane decreases the buckling pressure whereas a decrease of the tendons stiffness $E A_{t}$ increases the buckling pressure. The reasons for this are similar to those for the 145CA118-10-290 balloon. A linear interpolation of the power-laws for the single sensitivities results in the constants $a$ and $k$ as summarised in Table 3 .

\begin{tabular}{c|cccc} 
& Standard & $-10 \% E A_{t}$ & $-10 \% E_{m}$ & $-10 \% \nu$ \\
\hline$a$ & 6.2917 & 6.4033 & 6.1650 & 6.1180 \\
$k$ & -1.2733 & -1.3186 & -1.2421 & -1.2111
\end{tabular}

Table 3. Parameters $a$ and $k$ of power law for sensitivities of $n$ CR118-10-290, for 4up4down mode. 


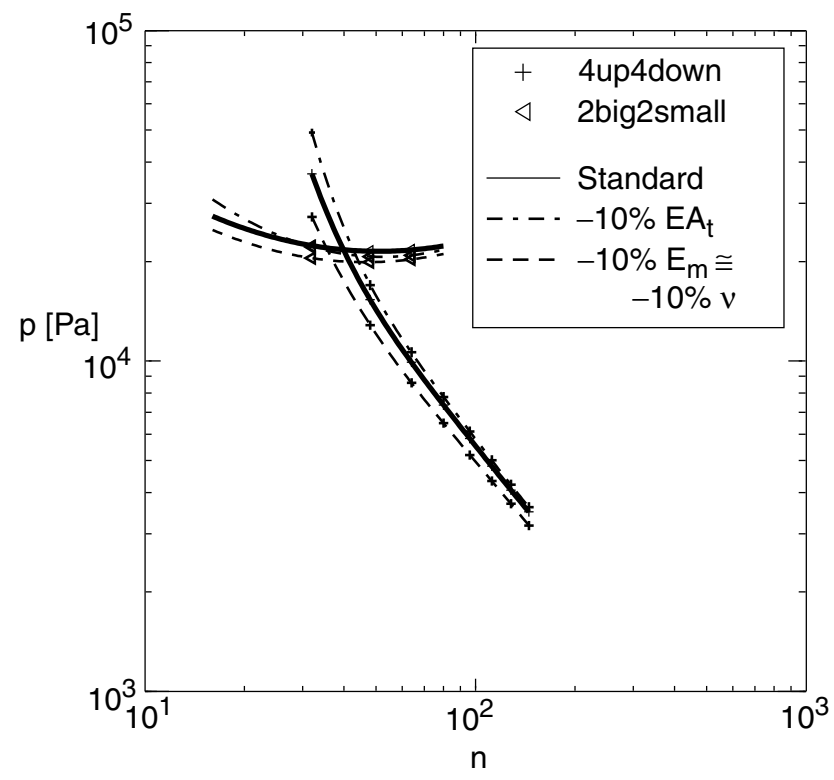

Figure 25. Material sensitivities of $n$ CR118-10-290 balloons.

\section{Discussion and Conclusion}

This paper has introduced a computational approach to the stability analysis of pumpkin balloons.

It has been shown how a single lobe is inflated from a flat cutting-pattern and how the eigenvalues and eigenvectors of a pumpkin balloon can be computed exploiting its symmetry, from the stiffness matrix for a single lobe. Detailed results have been given for balloons that are constructed from CA (constant angle) and CR (constant radius) cutting-patterns.

A key result of the paper is that the buckling pressure of a pumpkin balloon, that is a function of the number of lobes $n$, can be expressed by a power law and the sensitivity of the critical buckling pressure to different design parameters has been determined.

\section{Acknowledgements}

This research was partially supported by the NASA Balloon Program Office (BPO) and the NASA National Scientific Balloon Facility, Program Monitor Mr Danny Ball. MEP acknowledges financial support from EPSRC, Corpus Christi College and the Cambridge European Trust.

The authors thank Professor C.R. Calladine, Dr S.D. Guest, and Dr D. Wakefield for many helpful discussions.

\section{References}

${ }^{1}$ Taylor, G.I., On the Shapes of Parachutes, The Scientific Papers of G.I. Taylor, Cambridge University Press, 1963.

${ }^{2}$ Nott, J., Design Considerations and Practical Results with Long Duration Systems for Manned World Flights, Advances in Space Research, 2004, 33, 1667-1673.

${ }^{3}$ Calladine, C.R., Stability of the Endeavour balloon, Buckling of Structures, 1988, 133-149.

${ }^{4}$ Lennon, B.A., and Pellegrino S., Stability of Lobed Inflatable Structures, 41st AIAA/ASME/ASCE/AHS/ASC SDM Conference, AIAA 2000-1728, 2000.

${ }^{5}$ Smith, I.S., The NASA balloon program: looking to the future, Advances in Space Research, 33, 1588-1593, 2004.

${ }^{6}$ Schur, W.W., Jenkins, C.H. Deployment destiny, stable equilibria, the implications for Gossamer design, 43rd AIAA/ASME/ AHS/ASC Structural Dynamics, and Materials Conference Exhibit, Denver, CO, AIAA-2002-1205, April 2002.

${ }^{7}$ Smith, M.S, and Rainwater, E.L., Optimum designs for superpressure balloons, Advances in Space Research, 33, 16881693, 2004.

${ }^{8}$ Schur, W.W., Experimental investigation of undesired stable equilibria in pumpkin shape super-pressure balloon designs, Advances in Space Research, 33, 1682-1687, 2004. 
${ }^{9}$ Paulsen, W. H., What Is the Shape of a Mylar Balloon?, American Mathematical Monthly, 101, 953-958, 1994.

${ }^{10}$ Mladenov, I. M., and Oprea J., The Mylar Balloon Revisited, American Mathematical Monthly, 110, 761-784, 2003.

${ }^{11}$ Baginski, F. and Ramamurti, S., Variational principles for ascent shapes of large scientific balloons. AIAA Journal, 33, 764-768, 1995.

${ }^{12}$ Matlab 7, TheMathWorks, 3 Apple Hill Drive, Natick, MA 01760-2098.

${ }^{13}$ Schweizerhof, K., and Ramm, E., Displacement Dependend Pressure Loads in Non-Linear Finite Element Analysis, Computers\&Structures, 1984, 18, 1099-1114.

${ }^{14}$ Pagitz, M.E., and James, J., Symmetry Transformation Matrices, To be submitted.

${ }^{15}$ Felippa, C., Nonlinear Finite Element Methods, Lecture notes.

${ }^{16}$ Calladine, C.R., Theory of Shell Structures, Cambridge University Press, 1983. 